A.M. Escobar-Ruiz, J.C. López Vieyra, P. Winternitz and İ. Yurduşen

\title{
Fourth-order superintegrable systems separating in Polar Coordinates. II. Standard Potentials
}

\author{
Adrian M. Escobar-Ruiz, ${ }^{1, a)}$ J. C. López Vieyra, 2,b) P. Winternitz, ${ }^{1, \mathrm{c})}$ and \\ i. Yurdușen ${ }^{1,3, d)}$ \\ 1) Centre de recherches mathématiques and Département de mathématiques \\ et de statistique, Université de Montreal, C.P. 6128, succ. Centre-ville, \\ Montréal (QC) H3C 3J\%, Canada \\ ${ }^{2)}$ Instituto de Ciencias Nucleares, Universidad Nacional Autónoma de México, \\ Apartado Postal 70-543, 04510 México, D.F., Mexico \\ 3) Department of Mathematics, Hacettepe University, 06800 Beytepe, \\ Ankara, Turkey
}

(Dated: 11 May 2018)

Superintegrable Hamiltonian systems in a two-dimensional Euclidean space are considered. We present all real standard potentials that allow separation of variables in polar coordinates and admit an independent fourth-order integral of motion. The general form of the potentials satisfies a linear ODE. In the classical case, the standard potentials coincide with the Tremblay-Turbiner-Winternitz (TTW) or PostWinternitz (PW) models. In the quantum case new superintegrable systems are obtained, in addition to the TTW and PW ones. Their classical limit is free motion. Keywords: Superintegrability, separation of variables

\footnotetext{
a) Electronic mail: escobarr@crm.umontreal.ca

b) Electronic mail: vieyra@nucleares.unam.mx

c)Electronic mail: wintern@crm.umontreal.ca

d)Electronic mail: yurdusen@hacettepe.edu.tr
} 


\section{INTRODUCTION}

The purpose of this paper is to obtain and classify all classical and quantum superintegrable Hamiltonians that allow separation of variables in polar coordinates and admit a fourth-order integral of motion $Y$. We focus on standard potentials, namely those that satisfy (not trivially) a linear ODE. This linear ODE is nothing but the compatibility condition for the existence of the integral $Y$. Superintegrable systems separating in polar coordinates, allowing fourth-order integrals of motion and involving exotic potentials were studied in an earlier article $\frac{12}{2}$.

Roughly speaking, a Hamiltonian with $n$ degrees of freedom is called integrable if it allows $n$ independent well defined integrals of motion in involution. It is minimally superintegrable if it allows $n+1$ such integrals, and maximally superintegrable if it admits $2 n-1$ integrals (where only subsets of $n$ integrals among them can be in involution).

The best known superintegrable systems are the harmonic oscillator with its $s u(n+$ 1) algebra of integrals, and the Kepler-Coulomb system with its $o(n+1)$ algebra (when restricted to fixed bound state energy values).

A more recent review article provides precise and detailed definitions, general settings and motivation for studying superintegrable systems ${ }^{39}$. A systematic search for superintegrable classical and quantum systems in $E_{2}$ and $E_{3}$ established an interesting connection between second-order superintegrability and multiseparability in the Schrödinger or Hamilton-Jacobi equation $, \frac{5}{6}, 15,16,31,33,34$.

An extensive literature exists on second-order superintegrability in spaces of 2, 3 and $n$ dimensions, Riemannian and pseudo-Riemannian, real or complex, $\underline{24}-\underline{\underline{28}}, \underline{33}$.

The systematic study of higher order integrability is more recent. Pioneering work is due

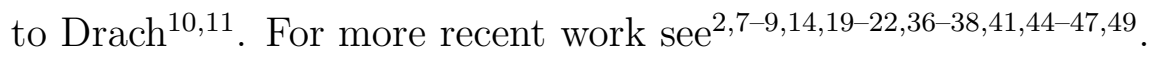

The present article is a contribution to a series $1,12,18,19,35,38,40,43,48$ devoted to superintegrable systems in $E_{2}$ with one integral of order $n \geq 3$ and one of order $n \leq 2$.

In this article we restrict ourselves to the space $E_{2}$. The Hamiltonian has the form

$$
H=\frac{1}{2}\left(p_{x}^{2}+p_{y}^{2}\right)+V(x, y)
$$

$V(x, y)$ is a scalar potential and in classical mechanics $p_{x}$ and $p_{y}$ are the momenta conjugate to the Cartesian coordinates $x$ and $y$. In quantum mechanics they are the corresponding 
operators $p_{x}=-i \hbar \frac{\partial}{\partial x}, p_{y}=-i \hbar \frac{\partial}{\partial y}$. In polar coordinates $(x, y) \equiv(r \cos \theta, r \sin \theta)$, the classical Hamiltonian reads

$$
H=\frac{1}{2}\left(p_{r}^{2}+\frac{p_{\theta}^{2}}{r^{2}}\right)+V(r, \theta)
$$

where $p_{r}$ and $p_{\theta}$ are the associated canonical momenta. The corresponding quantum operator takes the form

$$
H=-\frac{\hbar^{2}}{2}\left(\partial_{r}^{2}+\frac{1}{r} \partial_{r}+\frac{1}{r^{2}} \partial_{\theta}^{2}\right)+V(r, \theta)
$$

In paper ${ }^{12}$ we found all the exotic potentials, i.e. those which do not satisfy any linear differential equation. Here we solve the complementary problem, namely we find all the standard potentials which do satisfy a linear ODE. In all equations we keep the Planck constant $\hbar$ explicitly. Classical standard potentials will be obtained in the limit $\hbar \rightarrow 0$.

Before going into the question of standard superintegrable potentials with a fourth-order integral of motion, let us recall that two families of standard superintegrable potentials in $E_{2}$ are already known. Both allow separation of variables in polar coordinates and hence admit the second-order integral

$$
X=p_{\theta}^{2}+2 S(\theta), \quad p_{\theta}=L_{z}=-i \hbar \frac{\partial}{\partial \theta} .
$$

They also admit a further polynomial integral of arbitrary order $N$. One of them is the TTW potential

$$
V_{\mathrm{TTW}}=b r^{2}+\frac{1}{r^{2}}\left[\frac{\alpha}{\cos ^{2}(k \theta)}+\frac{\beta}{\sin ^{2}(k \theta)}\right],
$$

where $k=m / n$ and $m$ and $n$ are two integers (with no common divisors) $\underline{46.47}$. The other is the PW potential ${ }^{42}$

$$
V_{\mathrm{PW}}=\frac{a}{r}+\frac{1}{r^{2}}\left[\frac{\mu}{\cos ^{2}\left(\frac{k}{2} \theta\right)}+\frac{\nu}{\sin ^{2}\left(\frac{k}{2} \theta\right)}\right],
$$

where again $k=m / n$ is rational.

In the TTW case (5) the lowest order of an additional integral $Z$ was shown to be $\underline{17,29,30,44}$

$$
N=2(m+n-1)
$$

both in classical and quantum mechanics.

The TTW and PW potentials are related by coupling constant metamorphosis $, 23,32,42$. This transformation takes integrable and superintegrable systems into other systems that 
are also integrable or superintegrable, respectively. However, in general the order of the integrals of motion is not preserved, nor is their polynomial character. Special conditions ${ }^{32}$ must be satisfied for polynomial integrals to be transformed into polynomials of the same order. It has been conjectured and verified for $k=1$ and 2 that these conditions are satisfied for the TTW and PW systems $\frac{42}{2}$.

Thus we may expect that in any study of superintegrable systems separating in polar coordinates in $E_{2}$ with an additional integral of order $N$ with $2 \leq N<\infty$ the TTW and PW systems will appear as "standard" potentials.

A further comment is in order here and should be taken into account in any classification of superintegrable systems. If a certain potential $V(x, y)$ appears as superintegrable in $E_{2}$ with two integrals $X_{1}$ and $X_{2}$ that are polynomials of order $m$ and $n$, respectively, then the same potential will reappear at higher orders. Indeed $X_{1} X_{2}+X_{2} X_{1}$ will be an integral of order $m+n$ for the same Hamiltonian. The commutator $\left[X_{1}, X_{2}\right]$ (or Poisson bracket $\left.\left\{X_{1}, X_{2}\right\}_{\text {P.B. }}\right)$ will be a polynomial integral of order $m+n-1$. Such "trivially" superintegrable systems should be weeded out of lists of new superintegrable systems.

For fourth order superintegrable potentials, in addition to the Hamiltonian $H$, we have two more conserved quantities. A second-order integral (4), associated with the separation of variables in polar coordinates for which the potential takes the form

$$
V(r, \theta)=R(r)+\frac{S(\theta)}{r^{2}}
$$

and a fourth-order one

$$
\begin{aligned}
Y & =A_{1}\left\{L_{z}^{3}, p_{x}\right\}+A_{2}\left\{L_{z}^{3}, p_{y}\right\}+A_{3}\left\{L_{z}, p_{x}\left(p_{x}^{2}+p_{y}^{2}\right)\right\}+A_{4}\left\{L_{z}, p_{y}\left(p_{x}^{2}+p_{y}^{2}\right)\right\} \\
& +B_{1}\left(p_{x}^{4}-p_{y}^{4}\right)+2 B_{2} p_{x} p_{y}\left(p_{x}^{2}+p_{y}^{2}\right)+B_{3}\left\{L_{z}^{2}, p_{x}^{2}-p_{y}^{2}\right\}+2 B_{4}\left\{L_{z}^{2}, p_{x} p_{y}\right\} \\
& +C_{1}\left\{L_{z}, 3 p_{x}^{2} p_{y}-p_{y}^{3}\right\}+C_{2}\left\{L_{z}, p_{x}^{3}-3 p_{y}^{2} p_{x}\right\}+D_{1}\left(p_{x}^{4}+p_{y}^{4}-6 p_{x}^{2} p_{y}^{2}\right) \\
& +4 D_{2} p_{x} p_{y}\left(p_{x}^{2}-p_{y}^{2}\right)+\left\{g_{1}(x, y), p_{x}^{2}\right\}+\left\{g_{2}(x, y), p_{x} p_{y}\right\}+\left\{g_{3}(x, y), p_{y}^{2}\right\}+g_{4}(x, y)
\end{aligned}
$$

where the $A_{i}, B_{i}, C_{i}, D_{i}$ are real constants. The bracket $\{\cdot, \cdot\}$ denotes an anticommutator and $R(r), S(\theta), g_{1,2,3,4}(x, y)$ are real functions such that

$$
[H, Y]=[H, X]=0
$$

Under rotations around the $z$-axis, each of the six pairs of parameters in (9)

$$
\left(A_{1}, A_{2}\right),\left(A_{3}, A_{4}\right),\left(B_{1}, B_{2}\right),\left(B_{3}, B_{4}\right),\left(C_{1}, C_{2}\right),\left(D_{1}, D_{2}\right)
$$


forms a doublet. In general, $Y$ may contain the $O(2)$ singlets but by linear combinations of the form $Y+u_{1} X^{2}+u_{2} H^{2}+u_{3} X H$, where the $u_{i}$ are constants, we eliminated all such trivial terms. Under rotations through the angle $\theta$ the doublets $A_{i}, B_{i}, C_{i}, D_{i}$ rotate through $\theta, 2 \theta, 3 \theta, 4 \theta$, respectively.

We have $[Y, X]=C \neq 0$ where $C$ is in general a $5^{\text {th }}$-order linear operator. We thus obtain a finitely generated polynomial algebra of integrals of motion. We are looking for fourthorder superintegrable systems, so at least one of the constants $A_{i}, B_{i}, C_{i}, D_{i}$ is different from zero. The operator $Y$ is the most general polynomial expression for a fourth-order Hermitian operator of the required form. The commutator $[H, Y]$ contains derivatives of order up to three.

The operator $Y$ in (9) is given in Cartesian coordinates for brevity. Putting

$$
p_{x}=-i \hbar\left(\cos \theta \partial_{r}-\frac{\sin \theta}{r} \partial_{\theta}\right), \quad p_{y}=-i \hbar\left(\sin \theta \partial_{r}+\frac{\cos \theta}{r} \partial_{\theta}\right)
$$

we obtain the corresponding expression in polar coordinates. Explicitly, the leading terms of the integral $Y$ are

$$
\begin{aligned}
Y & =\hbar^{4}\left(\left(B_{1} \cos 2 \theta+B_{2} \sin 2 \theta+D_{1} \cos 4 \theta+D_{2} \sin 4 \theta\right) \partial_{r}^{4}+\frac{1}{r^{4}}\left[D_{2} \sin 4 \theta\right.\right. \\
& +D_{1} \cos 4 \theta-2 r\left(A_{1} r^{2}+A_{4}\right) \sin \theta-\left(B_{2}+2 B_{4} r^{2}\right) \sin 2 \theta+2 r\left(A_{2} r^{2}+A_{4}\right) \cos \theta \\
& \left.-\left(B_{1}+2 B_{3} r^{2}\right) \cos 2 \theta-2 r\left(C_{1} \cos 3 \theta-C_{2} \sin 3 \theta\right)\right] \partial_{\theta}^{4} \\
& -\frac{2}{r^{2}}\left[3\left(D_{1} \cos 4 \theta+D_{2} \sin 4 \theta\right)-r^{2}\left(B_{3} \cos 2 \theta+B_{4} \sin 2 \theta\right)\right. \\
& \left.+r\left(A_{3} \sin \theta-A_{4} \cos \theta-3\left(C_{1} \cos 3 \theta-C_{2} \sin 3 \theta\right)\right)\right] \partial_{r}^{2} \partial_{\theta}^{2} \\
& -\frac{2}{r}\left[B_{1} \sin 2 \theta-B_{2} \cos 2 \theta+2\left(D_{1} \sin 4 \theta-D_{2} \cos 4 \theta\right)-r\left(C_{1} \sin 3 \theta+C_{2} \cos 3 \theta\right.\right. \\
& \left.\left.+A_{3} \cos \theta+A_{4} \sin \theta\right)\right] \partial_{r}^{3} \partial_{\theta}-\frac{2}{r^{3}}\left[B_{1} \sin 2 \theta-B_{2} \cos 2 \theta\right. \\
& -2\left(D_{1} \sin 4 \theta+D_{2} \cos 4 \theta\right)-r\left(A_{3} \cos \theta+A_{4} \sin \theta-3\left(C_{1} \sin 3 \theta+C_{2} \cos 3 \theta\right)\right) \\
& \left.\left.+2 r^{2}\left(B_{3} \sin 2 \theta-B_{4} \cos 2 \theta\right)-r^{3}\left(A_{1} \cos \theta+A_{2} \sin \theta\right)\right] \partial_{r} \partial_{\theta}^{3}\right)+ \text { lower order terms } .
\end{aligned}
$$

For convenience let us introduce the functions

$$
G_{1}(r, \theta)=g_{1} \cos ^{2} \theta+g_{2} \sin ^{2} \theta+g_{3} \cos \theta \sin \theta
$$


A.M. Escobar-Ruiz, J.C. López Vieyra, P. Winternitz and İ. Yurduşen

$$
\begin{gathered}
G_{2}(r, \theta)=\frac{g_{1} \sin ^{2} \theta+g_{2} \cos ^{2} \theta-g_{3} \cos \theta \sin \theta}{r^{2}} \\
G_{3}(r, \theta)=-\frac{g_{1} \sin 2 \theta-g_{2} \sin 2 \theta-g_{3} \cos 2 \theta}{r} \\
G_{4}(r, \theta)=g_{4} .
\end{gathered}
$$

Then, we can write

$$
\begin{aligned}
& \left\{g_{1}(x, y), p_{x}^{2}\right\}+\left\{g_{2}(x, y), p_{x} p_{y}\right\}+\left\{g_{3}(x, y), p_{y}^{2}\right\}+g_{4}(x, y) \\
& =-\hbar^{2}\left(\left\{G_{1}(r, \theta), \partial_{r}^{2}\right\}+\left\{G_{3}(r, \theta), \partial_{r} \partial_{\theta}\right\}+\left\{G_{2}(r, \theta), \partial_{\theta}^{2}\right\}\right)+G_{4}(r, \theta)
\end{aligned}
$$

\section{DETERMINING EQUATIONS FOR A FOURTH-ORDER INTEGRAL}

\section{A. Commutator $[H, Y]$}

Since $Y$ is a fourth-order operator the commutator $[H, Y]$ would be a fifth-order one, i.e. we have

$$
[H, Y]=\sum_{k+l=0}^{N+1} Z_{k, l}(r, \theta) \frac{\partial^{k+l}}{\partial r^{k} \partial \theta^{l}}, \quad N=4
$$

and we require $Z_{k, l}=0$ for all $k$ and $l$. The terms of order $k+l=5$ and $k+l=4$ vanish automatically. It was already shown that for arbitrary $N$ the terms of order $N+1$ and $N$ $\operatorname{vanish}^{43}$. Moreover, only terms with $k+l$ of the opposite parity than $N$ provide independent

determining equations $\left(Z_{k, l}=0\right)$. Those with the same parity provide equations that are differential consequences of the first ones.

Finally, we obtain

$$
\begin{aligned}
{[H, Y] } & =\mathcal{A}_{r r r} \frac{\partial^{3}}{\partial r^{3}}+\mathcal{A}_{r r \theta} \frac{\partial^{3}}{\partial r^{2} \partial \theta}+\mathcal{A}_{r \theta \theta} \frac{\partial^{3}}{\partial r \partial \theta^{2}}+\mathcal{A}_{\theta \theta \theta} \frac{\partial^{3}}{\partial \theta^{3}} \\
& +\mathcal{C}_{r} \frac{\partial}{\partial r}+\mathcal{C}_{\theta} \frac{\partial}{\partial \theta}=0
\end{aligned}
$$

here the coefficients $\mathcal{A}_{r r r}, \mathcal{A}_{r r \theta}, \ldots$, are real functions of $r$ and $\theta$. The determining equations are obtained by setting all coefficients in (15) equal to zero. We thus obtain a total of 6 
PDEs for the functions $G_{1}, G_{2}, G_{3}, G_{4}, R$ and $S$. Four equations are independent of $\hbar$

$$
\begin{aligned}
G_{1}^{(1,0)} & =F_{1} R^{\prime}-\frac{2 F_{1}}{r^{3}} S+\frac{F_{2}}{r^{2}} S^{\prime}, \\
\frac{1}{r^{2}}\left(G_{2}^{(0,1)}+\frac{1}{r} G_{3}\right) & =F_{3} R^{\prime}-\frac{2 F_{3}}{r^{3}} S+\frac{F_{4}}{r^{2}} S^{\prime}, \\
\frac{1}{r^{2}} G_{1}^{(0,1)}+G_{3}^{(1,0)} & =3 F_{2} R^{\prime}-\frac{6 F_{2}}{r^{3}} S+\frac{F_{5}}{r^{2}} S^{\prime} \\
\frac{2}{r^{3}} G_{1}+G_{2}^{(1,0)}+\frac{1}{r^{2}} G_{3}^{(0,1)} & =F_{5} R^{\prime}-\frac{2 F_{5}}{r^{3}} S+\frac{3 F_{3}}{r^{2}} S^{\prime} .
\end{aligned}
$$

They are the same for classical or quantum systems, and two equations contain quantum corrections proportional to $\hbar^{2}$

$$
\begin{aligned}
& G_{3} \frac{1}{r^{2}} S^{\prime}+2 G_{1}\left(R^{\prime}-\frac{2}{r^{3}} S\right)-\frac{1}{2} G_{4}^{(1,0)} \\
& +\hbar^{2}\left[\frac{G_{1}^{(0,2)}}{2 r^{3}}-\frac{3 G_{3}^{(0,1)}}{4 r^{2}}+\frac{G_{3}^{(0,3)}}{4 r^{2}}-\frac{G_{1}^{(1,0)}}{r^{2}}-\frac{3}{2} G_{2}^{(1,0)}+\frac{5 G_{3}^{(1,1)}}{4 r}\right. \\
& \left.+\frac{G_{1}^{(1,2)}}{2 r^{2}}+\frac{1}{2} G_{2}^{(1,2)}+\frac{2 G_{1}^{(2,0)}}{r}-\frac{1}{2} r G_{2}^{(2,0)}+\frac{3}{4} G_{3}^{(2,1)}+G_{1}^{(3,0)}\right] \\
& =\hbar^{2}\left[\left(\frac{6 F_{6}}{r^{4}}-\frac{24 F_{1}}{r^{5}}-\frac{2 F_{11}}{r^{3}}\right) S+\left(\frac{6 F_{10}}{r^{4}}-\frac{4 F_{7}}{r^{3}}+\frac{F_{12}}{r^{2}}\right) S^{\prime}\right. \\
& \left.+\left(\frac{F_{8}}{r^{2}}-\frac{2 F_{5}}{r^{3}}\right) S^{\prime \prime}+\frac{F_{3}}{r^{2}} S^{\prime \prime \prime}+F_{11} R^{\prime}+F_{6} R^{\prime \prime}+F_{1} R^{\prime \prime \prime}\right] \\
& G_{3}\left(R^{\prime}-\frac{2}{r^{3}} S\right)+2 G_{2} \frac{1}{r^{2}} S^{\prime}-\frac{G_{4}{ }^{(0,1)}}{2 r^{2}} \\
& +\hbar^{2}\left[\frac{G_{3}}{4 r^{3}}-\frac{G_{2}{ }^{(0,1)}}{r^{2}}+\frac{5 G_{3}{ }^{(0,2)}}{4 r^{3}}+\frac{G_{2}{ }^{(0,3)}}{r^{2}}-\frac{G_{3}^{(1,0)}}{4 r^{2}}+\frac{G_{1}^{(1,1)}}{r^{3}}\right. \\
& \left.+\frac{3 G_{3}{ }^{(1,2)}}{4 r^{2}}+\frac{G_{3}{ }^{(2,0)}}{2 r}+\frac{G_{1}^{(2,1)}}{2 r^{2}}+\frac{1}{2} G_{2}^{(2,1)}+\frac{1}{4} G_{3}^{(3,0)}\right] \\
& =\hbar^{2}\left[\left(\frac{6 F_{7}}{r^{4}}-\frac{24 F_{2}}{r^{5}}-\frac{2 F_{12}}{r^{3}}\right) S+\left(\frac{6 F_{5}}{r^{4}}-\frac{4 F_{8}}{r^{3}}+\frac{F_{13}}{r^{2}}\right) S^{\prime}\right. \\
& \left.+\left(\frac{F_{9}}{r^{2}}-\frac{6 F_{3}}{r^{3}}\right) S^{\prime \prime}+\frac{F_{4}}{r^{2}} S^{\prime \prime \prime}+F_{12} R^{\prime}+F_{7} R^{\prime \prime}+F_{2} R^{\prime \prime \prime}\right] .
\end{aligned}
$$

The functions $F_{1} \ldots F_{13}$ are completely determined by the constants $A_{i}, B_{i}, C_{i}, D_{i}$ figuring in the leading part of the integral $Y$. They are presented in the Appendix.

The system of equations (16)-(21) is the main object of study of the present paper. 
A.M. Escobar-Ruiz, J.C. López Vieyra, P. Winternitz and İ. Yurduşen

\section{B. Linear compatibility condition}

The compatibility equation for (16)-(19) is a linear equation

$$
\begin{aligned}
& 0=\frac{1}{r^{2}}\left(16\left[A_{3} \cos \theta+A_{4} \sin \theta-9\left(C_{1} \sin 3 \theta+C_{2} \cos 3 \theta\right)\right] S\right. \\
& -20\left[A_{4} \cos \theta-A_{3} \sin \theta+9\left(C_{2} \sin 3 \theta-C_{1} \cos 3 \theta\right)\right] S^{\prime} \\
& +80\left[C_{1} \sin 3 \theta+C_{2} \cos 3 \theta\right] S^{\prime \prime} \\
& +5\left[A_{3} \sin \theta-A_{4} \cos \theta+3\left(C_{2} \sin 3 \theta-C_{1} \cos 3 \theta\right)\right] S^{(3)} \\
& \left.-\left[A_{3} \cos \theta+A_{4} \sin \theta+C_{1} \sin 3 \theta+C_{2} \cos 3 \theta\right] S^{(4)}\right) \\
& +\frac{1}{r^{3}}\left(96\left[B_{2} \cos 2 \theta-B_{1} \sin 2 \theta+8\left(D_{1} \sin 4 \theta-D_{2} \cos 4 \theta\right)\right] S\right. \\
& +40\left[B_{1} \cos 2 \theta+B_{2} \sin 2 \theta-20\left(D_{2} \sin 4 \theta+D_{1} \cos 4 \theta\right)\right] S^{\prime} \\
& +20\left[\left(B_{2} \cos 2 \theta-B_{1} \sin 2 \theta\right)+14\left(D_{2} \cos 4 \theta-D_{1} \sin 4 \theta\right)\right] S^{\prime \prime} \\
& +10\left[\left(B_{2} \sin 2 \theta+B_{1} \cos 2 \theta\right)+4\left(D_{1} \cos 4 \theta+D_{2} \sin 4 \theta\right)\right] S^{(3)} \\
& -\left[B_{2} \cos 2 \theta-B_{1} \sin 2 \theta+2\left(D_{2} \cos 4 \theta-D_{1} \sin 4 \theta\right] S^{(4)}\right) \\
& +\left[24 r^{3}\left(A_{1} \cos \theta+A_{2} \sin \theta\right)-12 r^{2}\left(B_{4} \cos 2 \theta-B_{3} \sin 2 \theta\right)\right. \\
& -r\left(2\left(A_{4} \sin \theta+A_{3} \cos \theta\right)+18\left(C_{1} \sin 3 \theta+C_{2} \cos 3 \theta\right)\right) \\
& \left.+3\left(B_{2} \cos 2 \theta-B_{1} \sin 2 \theta\right)+30\left(D_{2} \cos 4 \theta-D_{1} \sin 4 \theta\right)\right] R^{\prime} \\
& +\left[36 r^{4}\left(A_{1} \cos \theta+A_{2} \sin \theta\right)-12 r^{3}\left(B_{3} \sin 2 \theta-B_{4} \cos 2 \theta\right)\right. \\
& +r^{2}\left(2\left(A_{3} \cos \theta+A_{4} \sin \theta\right)+18\left(C_{1} \sin 3 \theta+C_{2} \cos 3 \theta\right)\right) \\
& \left.\left.+r\left(3\left(B_{1} \sin 2 \theta-B_{2} \cos 2 \theta\right)\right)-30\left(D_{2} \cos 4 \theta-D_{1} \sin 4 \theta\right)\right)\right] R^{\prime \prime} \\
& +\left[12 r^{5}\left(A_{1} \cos \theta+A_{2} \sin \theta\right)+14 r^{4}\left(B_{4} \cos 2 \theta-B_{3} \sin 2 \theta\right)\right. \\
& +r^{3}\left(5\left(A_{3} \cos \theta+A_{4} \sin \theta\right)-3\left(C_{1} \sin 3 \theta+C_{2} \cos 3 \theta\right)\right) \\
& \left.+12 r^{2}\left(D_{2} \cos 4 \theta-D_{1} \sin 4 \theta\right)\right] R^{(3)} \\
& +\left[r^{6}\left(A_{1} \cos \theta+A_{2} \sin \theta\right)+2 r^{5}\left(B_{4} \cos 2 \theta-B_{3} \sin 2 \theta\right)\right. \\
& +r^{4}\left(A_{4} \sin \theta+A_{3} \cos \theta-3\left(C_{1} \sin 3 \theta+C_{2} \cos 3 \theta\right)\right) \\
& \left.-r^{3}\left(B_{1} \sin 2 \theta-B_{2} \cos 2 \theta+2\left(D_{2} \cos 4 \theta-D_{1} \sin 4 \theta\right)\right)\right] R^{(4)},
\end{aligned}
$$

for the functions $R(r)$ and $S(\theta)$ in the potential. It is the same in classical and quantum mechanics (as are equations (16), ..., (19)). 
We will henceforth define exotic potentials as those for which the compatibility condition is satisfied trivially. For $N=4$ this means all the coefficients in the integral $Y$ of (9) except $A_{1}, A_{2}, B_{3}$ and $B_{4}$ vanish. Standard potentials must satisfy the linear PDE (22) nontrivially. Hence at least one of the constants $A_{i}, B_{i}, C_{i}$ or $D_{i}$ in (22) must be nonzero.

Differentiating (22) with respect to $r$ we eliminate $S(\theta)$ for the equation. Following paper $^{12}$ we obtain 8 ODEs for $R(r)$ by setting the coefficients of $\cos k \theta$ and $\sin k \theta$ for $k=$ 1,2,3,4 equal to zero. These together with the determining equations imply that the only possible forms of $R(r)$ are

$$
R(r)=0, \quad R(r)=\frac{a}{r}, \quad R(r)=b r^{2} .
$$

The result (23) is actually a consequence of Bertrand's theorem ${ }^{3}$ and is valid for integrals of motion $Y$ of any order $\stackrel{13}{ }$. Let us now consider each of the cases listed in (23) separately.

\section{NON CONFINING POTENTIAL $V(r, \theta)=\frac{S(\theta)}{r^{2}}$}

Here we address the case of a non confining potential, namely $R(r)=0$. It turns out that this case provides the most general form of the function $S(\theta)$. The same function $S(\theta)$ will reoccur for $R(r) \neq 0$.

The equations (16), (18) and (19) corresponding to the determining equations $\mathcal{A}_{r r r}=$ $\mathcal{A}_{r r \theta}=\mathcal{A}_{r \theta \theta}=0$, respectively, take the following form

$$
\begin{aligned}
& G_{1}^{(1,0)}(r, \theta) r^{3}-\left(\left(A_{3} \cos \theta+A_{4} \sin \theta+C_{1} \sin 3 \theta+C_{2} \cos 3 \theta\right) r\right. \\
& \left.-\left(B_{1} \sin 2 \theta-B_{2} \cos 2 \theta+2\left(D_{1} \sin 4 \theta-D_{2} \cos 4 \theta\right)\right)\right) S^{\prime} \\
& +4\left(B_{1} \cos 2 \theta+B_{2} \sin 2 \theta+D_{1} \cos 4 \theta+D_{2} \sin 4 \theta\right) S=0 \\
& G_{3}^{(1,0)}(r, \theta) r^{4}+G_{1}^{(0,1)}(r, \theta) r^{2}-2\left(\left(B_{3} \cos 2 \theta+B_{4} \sin 2 \theta\right) r^{2}\right. \\
& -\left(A_{3} \sin \theta-A_{4} \cos \theta-3\left(C_{1} \cos 3 \theta-C_{2} \sin 3 \theta\right)\right) r \\
& \left.-3\left(D_{1} \cos 4 \theta+D_{2} \sin 4 \theta\right)\right) S^{\prime} \\
& +6\left(\left(A_{3} \cos \theta+A_{4} \sin \theta+C_{1} \sin 3 \theta+C_{2} \cos 3 \theta\right) r\right. \\
& \left.-\left(B_{1} \sin 2 \theta-B_{2} \cos 2 \theta+2\left(D_{1} \sin 4 \theta-D_{2} \cos 4 \theta\right)\right)\right) S=0
\end{aligned}
$$


A.M. Escobar-Ruiz, J.C. López Vieyra, P. Winternitz and İ. Yurduşen

$$
\begin{aligned}
& G_{2}^{(1,0)}(r, \theta) r^{5}+G_{3}^{(0,1)}(r, \theta) r^{3}+2 G_{1}(r, \theta) r^{2} \\
& -3\left(\left(A_{1} \cos \theta+A_{2} \sin \theta\right) r^{3}+2\left(B_{4} \cos 2 \theta-B_{3} \sin 2 \theta\right) r^{2}\right. \\
& +\left(A_{3} \cos \theta+A_{4} \sin \theta-3\left(C_{1} \sin 3 \theta+C_{2} \cos 3 \theta\right)\right) r \\
& \left.-B_{1} \sin 2 \theta+B_{2} \cos 2 \theta+2\left(D_{1} \sin 4 \theta-D_{2} \cos 4 \theta\right)\right) S^{\prime} \\
& +4\left(\left(B_{3} \cos 2 \theta+B_{4} \sin 2 \theta\right) r^{2}\right. \\
& +\left(A_{4} \cos \theta-A_{3} \sin \theta+3\left(C_{1} \cos 3 \theta-C_{2} \sin 3 \theta\right)\right) r \\
& \left.-3\left(D_{1} \cos 4 \theta+D_{2} \sin 4 \theta\right)\right) S=0 .
\end{aligned}
$$

The above equations can be integrated to find the functions $G_{1}(r, \theta), G_{3}(r, \theta)$ and $G_{2}(r, \theta)$ in terms of $S(\theta)$. They are given by the following formulas:

$$
\begin{aligned}
G_{1}(r, \theta)= & \left(\frac{1}{2 r^{2}}\left(B_{1} \sin 2 \theta-B_{2} \cos 2 \theta+2\left(D_{1} \sin 4 \theta-D_{2} \cos 4 \theta\right)\right)\right. \\
& \left.-\frac{1}{r}\left(A_{3} \cos \theta+A_{4} \sin \theta+C_{1} \sin 3 \theta+C_{2} \cos 3 \theta\right)\right) S^{\prime} \\
& +\frac{2}{r^{2}}\left(B_{1} \cos 2 \theta+B_{2} \sin 2 \theta+D_{1} \cos 4 \theta+D_{2} \sin 4 \theta\right) S+\gamma_{1}(\theta) \\
G_{3}(r, \theta)= & -\frac{2}{r}\left(B_{3} \cos 2 \theta+B_{4} \sin 2 \theta\right) S^{\prime}+\frac{1}{r^{2}}\left[3\left(A_{3} \cos \theta+A_{4} \sin \theta+C_{1} \sin 3 \theta+C_{2} \cos 3 \theta\right) S\right. \\
+ & \frac{3}{2}\left(\left(A_{3} \sin \theta-A_{4} \cos \theta\right)-3\left(C_{1} \cos 3 \theta-C_{2} \sin 3 \theta\right)\right) S^{\prime} \\
- & \left.\frac{1}{2}\left(\left(A_{3} \cos \theta+A_{4} \sin \theta\right)+\left(C_{1} \sin 3 \theta+C_{2} \cos 3 \theta\right)\right) S^{\prime \prime}\right] \\
+ & \frac{1}{r^{3}}\left[-\frac{10}{3}\left(\left(B_{1} \sin 2 \theta-B_{2} \cos 2 \theta\right)+2\left(D_{1} \sin 4 \theta-D_{2} \cos 4 \theta\right)\right) S\right. \\
+ & \left(B_{1} \cos 2 \theta+B_{2} \sin 2 \theta+4\left(D_{1} \cos 4 \theta+D_{2} \sin 4 \theta\right)\right) S^{\prime} \\
+ & \left.\frac{1}{6}\left(B_{1} \sin 2 \theta-B_{2} \cos 2 \theta+2\left(D_{1} \sin 4 \theta-D_{2} \cos 4 \theta\right)\right) S^{\prime \prime}\right] \\
+ & \frac{1}{r} \gamma_{1}^{\prime}(\theta)+\gamma_{3}(\theta),
\end{aligned}
$$




$$
\begin{aligned}
G_{2}(r, \theta) & =-\frac{3}{r}\left(A_{1} \cos \theta+A_{2} \sin \theta\right) S^{\prime}+\frac{1}{r^{2}}\left[\left(2\left(B_{3} \cos 2 \theta+B_{4} \sin 2 \theta\right)\right) S\right. \\
& \left.+5\left(B_{3} \sin 2 \theta-B_{4} \cos 2 \theta\right) S^{\prime}-\left(B_{3} \cos 2 \theta+B_{4} \sin 2 \theta\right) S^{\prime \prime}\right] \\
& +\frac{1}{r^{3}}\left[-\frac{7}{3}\left(\left(A_{3} \sin \theta-A_{4} \cos \theta\right)-3\left(C_{1} \cos 3 \theta-C_{2} \sin 3 \theta\right)\right) S\right. \\
& -\frac{1}{6}\left(\left(A_{3} \cos \theta+A_{4} \sin \theta\right)-47\left(C_{1} \sin 3 \theta+C_{2} \cos 3 \theta\right)\right) S^{\prime} \\
& +\frac{2}{3}\left(\left(A_{3} \sin \theta-A_{4} \cos \theta\right)-3\left(C_{1} \cos 3 \theta-C_{2} \sin 3 \theta\right)\right) S^{\prime \prime} \\
& \left.-\frac{1}{6}\left(\left(A_{3} \cos \theta+A_{4} \sin \theta\right)+\left(C_{1} \sin 3 \theta+C_{2} \cos 3 \theta\right)\right) S^{\prime \prime \prime}\right] \\
& +\frac{1}{r^{4}}\left[-\frac{2}{3}\left(\left(B_{1} \cos 2 \theta+B_{2} \sin 2 \theta\right)+13\left(D_{1} \cos 4 \theta+D_{2} \sin 4 \theta\right)\right) S\right. \\
& -\frac{1}{3}\left(\left(B_{1} \sin 2 \theta-B_{2} \cos 2 \theta\right)+20\left(D_{1} \sin 4 \theta-D_{2} \cos 4 \theta\right)\right) S^{\prime} \\
& +\frac{1}{3}\left(\left(B_{1} \cos 2 \theta+B_{2} \sin 2 \theta\right)+4\left(D_{1} \cos 4 \theta+D_{2} \sin 4 \theta\right)\right) S^{\prime \prime} \\
& \left.+\frac{1}{24}\left(\left(B_{1} \sin 2 \theta-B_{2} \cos 2 \theta\right)+2\left(D_{1} \sin 4 \theta-D_{2} \cos 4 \theta\right)\right) S^{\prime \prime \prime}\right] \\
& +\frac{1}{r} \gamma_{3}^{\prime}(\theta)+\frac{1}{r^{2}} \gamma_{1}(\theta)+\frac{1}{2 r^{2}} \gamma_{1}^{\prime \prime}(\theta)+\gamma_{2}(\theta),
\end{aligned}
$$

where $\gamma_{1}(\theta), \gamma_{2}(\theta)$ and $\gamma_{3}(\theta)$ are functions of $\theta$ still to be determined.

The determining equation $\mathcal{A}_{\theta \theta \theta}=0$ (17) has not been used yet. We now substitute the expressions (27)-(29) into (17) in order to obtain further information on functions of $\theta$, namely $S(\theta)$ and $\gamma_{k}(\theta), k=1,2,3$. The variable $r$ still figures in the obtained result but only explicitly as $r^{k}, k=0,1,2,3,4$ (no unknown functions of $r$ remain). Thus, the coefficient of $r^{k}$ in $\mathcal{A}_{\theta \theta \theta}=0$ (17) must vanish for each $k$ and we obtain a system of 5 equations for the 4 remaining functions of $\theta$. They are

$$
\begin{aligned}
& 0=\gamma_{2}^{\prime} \\
& 0=4\left(A_{1} \cos \theta+A_{2} \sin \theta\right) S+14\left(A_{1} \sin \theta-A_{2} \cos \theta\right) S^{\prime} \\
& -6\left(A_{1} \cos \theta+A_{2} \sin \theta\right) S^{\prime \prime}+2 \gamma_{3}^{\prime \prime}+2 \gamma_{3}, \\
& 0=-16\left(B_{3} \sin 2 \theta-B_{4} \cos 2 \theta\right) S+28\left(B_{3} \cos 2 \theta+B_{4} \sin 2 \theta\right) S^{\prime} \\
& +14\left(B_{3} \sin 2 \theta-B_{4} \cos 2 \theta\right) S^{\prime \prime}-2\left(B_{3} \cos 2 \theta+B_{4} \sin 2 \theta\right) S^{(3)} \\
& +4 \gamma_{1}^{\prime}+\gamma_{1}^{(3)}
\end{aligned}
$$




$$
\begin{aligned}
0 & =96\left(\left(B_{2} \cos 2 \theta-B_{1} \sin 2 \theta\right)+8\left(D_{1} \sin 4 \theta-D_{2} \cos 4 \theta\right)\right) S \\
+ & 40\left(\left(B_{1} \cos 2 \theta+B_{2} \sin 2 \theta\right)-20\left(D_{1} \cos 4 \theta+D_{2} \sin 4 \theta\right)\right) S^{\prime} \\
+ & 20\left(\left(B_{2} \cos 2 \theta-B_{1} \sin 2 \theta\right)+14\left(D_{2} \cos 4 \theta-D_{1} \sin 4 \theta\right)\right) S^{\prime \prime} \\
+ & 10\left(\left(B_{1} \cos 2 \theta+B_{2} \sin 2 \theta\right)+4\left(D_{1} \cos 4 \theta+D_{2} \sin 4 \theta\right)\right) S^{(3)} \\
+ & \left(\left(B_{1} \sin 2 \theta-B_{2} \cos 2 \theta\right)+2\left(D_{1} \sin 4 \theta-D_{2} \cos 4 \theta\right)\right) S^{(4)} \\
& =16\left(\left(A_{3} \cos \theta+A_{4} \sin \theta\right)-9\left(C_{1} \sin 3 \theta+C_{2} \cos 3 \theta\right)\right) S \\
& +20\left(\left(A_{3} \sin \theta-A_{4} \cos \theta\right)+9\left(C_{1} \cos 3 \theta-C_{2} \sin 3 \theta\right)\right) S^{\prime} \\
& +80\left(C_{1} \sin 3 \theta+C_{2} \cos 3 \theta\right) S^{\prime \prime} \\
& +5\left(\left(A_{3} \sin \theta-A_{4} \cos \theta\right)-3\left(C_{1} \cos 3 \theta-C_{2} \sin 3 \theta\right)\right) S^{(3)} \\
& -\left(A_{3} \cos \theta+A_{4} \sin \theta+C_{1} \sin 3 \theta+C_{2} \cos 3 \theta\right) S^{(4)}
\end{aligned}
$$

Let us now discuss these equations.

1. The equation (30) determines $\gamma_{2}=$ constant.

2. Equations (31) and (32) must be used to determine $\gamma_{1}(\theta)$ and $\gamma_{3}(\theta)$ in terms of the function $S(\theta)$ figuring in the potential.

3. Of the 12 "doublet coefficients" figuring in the leading part of the integral $Y$, four figure only in (33), namely $B_{1}, B_{2}, D_{1}, D_{2}$ and four only in (34), namely $A_{3}, A_{4}, C_{1}, C_{2}$. The remaining ones $\left(A_{1}, A_{2}\right)$ and $\left(B_{3}, B_{4}\right)$ appear only in (31) and (32), respectively. By definition, the linear compatibility condition (22) must be satisfied trivially for exotic potentials at $R(r)=0$. Hence, for these potentials the only possible constants figuring in $Y$ are $\left(A_{1}, A_{2}\right)$ and $\left(B_{3}, B_{4}\right)$. This case was analyzed conclusively and in detail in our previous article $\frac{12}{2}$.

Let us now turn to the problem of finding all standard superintegrable potentials. To do this we proceed as follows

- I. Assume that at least one of the constants $B_{1}, B_{2}, D_{1}, D_{2}$ is not vanishing and solve (33) for $S(\theta)$. The general solution for $S(\theta)$ will depend on these constants and on additional integration constants. 
- II. We proceed similarly with equation (34), i.e. solve it assuming that at least one of the $A_{3}, A_{4}, C_{1}, C_{2}$ is non-zero.

In both cases it is still necessary to solve the determining equations

$$
\mathcal{C}_{r}=0, \quad \mathcal{C}_{\theta}=0
$$

with $\mathcal{C}_{r}$ and $\mathcal{C}_{\theta}$ figuring in (15), (see (20)-(21) $)$. The determining equation $\mathcal{C}_{r}=0$ defines $G_{4}(r, \theta), \mathcal{C}_{\theta}=0$ will provide further information and make it possible to determine $S(\theta)$ completely and hence also the integrals $X$ and $Y$.

- III. It is also possible for both of the above sets of constants to contain nonzero constants. The corresponding potentials will then allow more than one fourth-order integral. However, at most 3 integrals $(H, X$ and $Y$ ) can be polynomially independent. In any case, the function $S_{I I I}(\theta)$ obtained in this case will be a special case of both $S_{I}$ and $S_{I I}$ and we shall not pursue this issue further.

\section{A. General form of the angular part $S(\theta)$}

Case I. $\left(B_{1}, B_{2}, D_{1}, D_{2}\right) \neq(0,0,0,0)$

We concentrate in equation (33). We first define $T_{I}(\theta)$ by putting

$$
T_{I}^{\prime}(\theta) \equiv S_{I}(\theta)
$$

The general solution of the fifth-order linear ODE for $T_{I}$ obtained from (33) is

$$
T_{I}(\theta)=\frac{1}{M_{I}}\left(s_{1}+s_{2} \sin 2 \theta+s_{3} \cos 2 \theta+s_{4} \sin 4 \theta+s_{5} \cos 4 \theta\right)
$$

where the $s_{i}$ are integration constants and

$$
M_{I}=B_{1} \sin 2 \theta-B_{2} \cos 2 \theta+2\left(D_{1} \sin 4 \theta-D_{2} \cos 4 \theta\right) .
$$


A.M. Escobar-Ruiz, J.C. López Vieyra, P. Winternitz and İ. Yurduşen

Using (36) we obtain $S_{I}(\theta)$ as

$$
\begin{aligned}
S_{I}(\theta)= & -\frac{1}{M_{I}^{2}}\left(2 s_{1}\left[B_{1} \cos 2 \theta+B_{2} \sin 2 \theta+4\left(D_{1} \cos 4 \theta+D_{2} \sin 4 \theta\right)\right]\right. \\
& +2 s_{2}\left[B_{1}+D_{1} \cos 6 \theta+D_{2} \sin 6 \theta+3\left(D_{1} \cos 2 \theta+D_{2} \sin 2 \theta\right)\right] \\
& +2 s_{3}\left[B_{2}-D_{2} \cos 6 \theta+D_{1} \sin 6 \theta+3\left(D_{2} \cos 2 \theta-D_{1} \sin 2 \theta\right)\right] \\
& +s_{4}\left[8 D_{1}-B_{1} \cos 6 \theta-B_{2} \sin 6 \theta+3\left(B_{1} \cos 2 \theta-B_{2} \sin 2 \theta\right)\right] \\
+ & \left.s_{5}\left[8 D_{2}+B_{2} \cos 6 \theta-B_{1} \sin 6 \theta+3\left(B_{2} \cos 2 \theta+B_{1} \sin 2 \theta\right)\right]\right) .
\end{aligned}
$$

There seem to be too many integration constants in (38), since (33) is a fourth-order ODE for $S(\theta)$. Indeed the five solutions corresponding to $s_{1}, \ldots, s_{5}$, are not independent and one can be eliminated in terms of the others. We shall not go into this here since constraints on the constants $s_{i}$ will be imposed by the last still unsolved determining equation, namely $\mathcal{C}_{\theta}=0$ and these will always leave less than 5 free constants.

Case II $\left(A_{3}, A_{4}, C_{1}, C_{2}\right) \neq(0,0,0,0)$

Similarly, the solution to the second linear equation (34) is

$$
T_{I I}(\theta)=\frac{1}{M_{I I}}\left(s_{1}+s_{2} \sin \theta+s_{3} \cos \theta+s_{4} \sin 3 \theta+s_{5} \cos 3 \theta\right),
$$

where

$$
M_{I I}=A_{3} \cos \theta+A_{4} \sin \theta+C_{1} \sin 3 \theta+C_{2} \cos 3 \theta .
$$

Consequently

$$
\begin{aligned}
S_{I I}(\theta) & =\frac{1}{M_{I I}^{2}}\left(2 s_{1}\left[A_{3} \sin \theta-A_{4} \cos \theta+3\left(C_{2} \sin 3 \theta-C_{1} \cos 3 \theta\right)\right]\right. \\
& +s_{2}\left[A_{3}-C_{2} \cos 4 \theta-C_{1} \sin 4 \theta+2\left(C_{2} \cos 2 \theta+C_{1} \sin 2 \theta\right)\right] \\
& -s_{3}\left[A_{4}+C_{1} \cos 4 \theta-C_{2} \sin 4 \theta+2\left(C_{1} \cos 2 \theta-C_{2} \sin 2 \theta\right)\right] \\
+ & s_{4}\left[3 C_{2}+A_{3} \cos 4 \theta+A_{4} \sin 4 \theta+2\left(A_{3} \cos 2 \theta-A_{4} \sin 2 \theta\right)\right] \\
- & \left.s_{5}\left[3 C_{1}-A_{4} \cos 4 \theta+A_{3} \sin 4 \theta+2\left(A_{4} \cos 2 \theta+A_{3} \sin 2 \theta\right)\right]\right) .
\end{aligned}
$$

Again the five solutions corresponding to the 5 constants $s_{i}$, are not linearly independent.

As mentioned above, the determining equation $\mathcal{C}_{r}=0(20)$ defines the function $G_{4}(r, \theta)$ in both cases. The last equation $\mathcal{C}_{\theta}=0$ (21) constrains the coefficients $s_{i}$ in both cases. We again treat cases I and II, separately. 
A.M. Escobar-Ruiz, J.C. López Vieyra, P. Winternitz and İ. Yurduşen

Case I $\left(B_{1}, B_{2}, D_{1}, D_{2}\right) \neq(0,0,0,0)$

In this case, from the equation $\mathcal{C}_{r}=0(20)$ we obtain the function $G_{4}(r, \theta)$

$$
\begin{aligned}
48 r^{4} G_{4}(r, \theta) & =4\left(32 S S^{\prime}\left(B_{1} \sin 2 \theta-B_{2} \cos 2 \theta+2\left(D_{1} \sin 4 \theta-D_{2} \cos 4 \theta\right)\right)\right. \\
& +48 S^{2}\left(B_{1} \cos 2 \theta+B_{2} \sin 2 \theta+D_{1} \cos 4 \theta+D_{2} \sin 4 \theta\right) \\
& +S^{\prime}\left[S^{\prime \prime}\left(B_{2} \cos 2 \theta-B_{1} \sin 2 \theta-2\left(D_{1} \sin 4 \theta-D_{2} \cos 4 \theta\right)\right)\right. \\
& \left.\left.-6 S^{\prime}\left(B_{1} \cos 2 \theta+B_{2} \sin 2 \theta+4\left(D_{1} \cos 4 \theta+D_{2} \sin 4 \theta\right)\right)\right]\right) \\
& +\hbar^{2}\left[3072 S\left(D_{1} \cos 4 \theta+D_{2} \sin 4 \theta\right)\right. \\
& +64 S^{\prime}\left(B_{1} \sin 2 \theta-B_{2} \cos 2 \theta+62\left(D_{1} \sin 4 \theta-D_{2} \cos 4 \theta\right)\right) \\
& -96 S^{\prime \prime}\left(B_{1} \cos 2 \theta+B_{2} \sin 2 \theta+20\left(D_{2} \sin 4 \theta+D_{1} \cos 4 \theta\right)\right) \\
& +S^{(3)}\left(52\left(B_{2} \cos 2 \theta-B_{1} \sin 2 \theta\right)+440\left(D_{2} \cos 4 \theta-D_{1} \sin 4 \theta\right)\right) \\
& +12 S^{(4)}\left(B_{1} \cos 2 \theta+B_{2} \sin 2 \theta+4\left(D_{2} \sin 4 \theta+D_{1} \cos 4 \theta\right)\right) \\
& \left.+S^{(5)}\left(B_{1} \sin 2 \theta-B_{2} \cos 2 \theta+2\left(D_{1} \sin 4 \theta-D_{2} \cos 4 \theta\right)\right)\right]
\end{aligned}
$$


A.M. Escobar-Ruiz, J.C. López Vieyra, P. Winternitz and İ. Yurduşen

The determining equation $\mathcal{C}_{\theta}=0$ reads

$$
\begin{aligned}
& 4\left(256 S^{2}\left(B_{1} \sin 2 \theta-B_{2} \cos 2 \theta+2\left(D_{1} \sin 4 \theta-D_{2} \cos 4 \theta\right)\right)\right. \\
& -240 S S^{\prime}\left(B_{2} \sin 2 \theta+B_{1} \cos 2 \theta+4\left(D_{1} \cos 4 \theta+D_{2} \sin 4 \theta\right)\right) \\
& +60\left(S^{\prime}\right)^{2}\left(B_{2} \cos 2 \theta-B_{1} \sin 2 \theta-8\left(D_{1} \sin 4 \theta-D_{2} \cos 4 \theta\right)\right) \\
& +40 S S^{\prime \prime}\left(B_{2} \cos 2 \theta-B_{1} \sin 2 \theta-2\left(D_{1} \sin 4 \theta-D_{2} \cos 4 \theta\right)\right) \\
& +30 S^{\prime} S^{\prime \prime}\left(B_{1} \cos 2 \theta+B_{2} \sin 2 \theta+4\left(D_{1} \cos 4 \theta+D_{2} \sin 4 \theta\right)\right) \\
& +\left(S^{\prime \prime}\right)^{2}\left(B_{1} \sin 2 \theta-B_{2} \cos 2 \theta+2\left(D_{1} \sin 4 \theta-D_{2} \cos 4 \theta\right)\right) \\
& \left.+3 S^{(3)} S^{\prime}\left(B_{1} \sin 2 \theta-B_{2} \cos 2 \theta+2\left(D_{1} \sin 4 \theta-D_{2} \cos 4 \theta\right)\right)\right) \\
& +\hbar^{2}\left[384 S\left(B_{2} \cos 2 \theta-B_{1} \sin 2 \theta-88\left(D_{1} \sin 4 \theta-D_{2} \cos 4 \theta\right)\right)\right. \\
& -32 S^{\prime}\left(31\left(B_{1} \cos 2 \theta+B_{2} \sin 2 \theta\right)-1676\left(D_{1} \cos 4 \theta+D_{2} \sin 4 \theta\right)\right) \\
& +16 S^{\prime \prime}\left(53\left(B_{2} \cos 2 \theta-B_{1} \sin 2 \theta\right)+2114\left(D_{1} \sin 4 \theta-D_{2} \cos 4 \theta\right)\right) \\
& -80 S^{(3)}\left(B_{1} \cos 2 \theta+B_{2} \sin 2 \theta+136\left(D_{1} \cos 4 \theta+D_{2} \sin 4 \theta\right)\right) \\
& -16 S^{(4)}\left(11\left(B_{1} \sin 2 \theta-B_{2} \cos 2 \theta\right)+118\left(D_{1} \sin 4 \theta-D_{2} \cos 4 \theta\right)\right) \\
& +42 S^{(5)}\left(B_{1} \cos 2 \theta+B_{2} \sin 2 \theta+4\left(D_{1} \cos 4 \theta+D_{2} \sin 4 \theta\right)\right) \\
& \left.+3 S^{(6)}\left(B_{1} \sin 2 \theta-B_{2} \cos 2 \theta+2\left(D_{1} \sin 4 \theta-D_{2} \cos 4 \theta\right)\right)\right]=0
\end{aligned}
$$


First, by an appropriate rotation we can always set $B_{1}=0$. Then, we substitute the function (38) in the remaining equation (42). As a result we obtain algebraic equations for the $s_{i}(i=1,2,3,4,5)$ in (38) by setting the coefficients of $\cos k \theta$ and $\sin k \theta$ for $k=$ $0,2,4, \ldots, 18$ equal to zero. We obtain the following solutions

$$
\begin{aligned}
& s_{1}^{(\ell)}=\frac{q_{\ell}}{4 D_{1}^{2} \hbar^{6}\left(B_{2}^{2}-8 D_{1}^{2}\right)^{2} D_{2}^{2}}\left[B_{2} D_{2}\left(8 D_{1}^{2}-B_{2}^{2}\right)\left(B_{2}^{2}\left(D_{1}^{2}+D_{2}^{2}\right)+8 D_{1}^{2}\left(4 D_{1}^{2}+3 D_{2}^{2}\right)\right) \hbar^{6}\right. \\
& +\left(8 B_{2}^{2}\left(D_{1}^{5}-6 D_{1}^{3} D_{2}^{2}\right)-B_{2}^{4}\left(D_{1}^{3}+3 D_{2}^{2} D_{1}\right)+64\left(2 D_{1}^{2}+D_{2}^{2}\right) D_{1}^{5}\right) \hbar^{4} q_{\ell} \\
& \left.B_{2} D_{1}^{2} D_{2}\left(3 B_{2}^{2}+40 D_{1}^{2}\right) \hbar^{2} q_{\ell}^{2}-D_{1}^{3}\left(B_{2}^{2}+8 D_{1}^{2}\right) q_{\ell}^{3}\right], \\
& s_{2}^{(\ell)}=q_{\ell} \hbar^{2}, \\
& s_{3}^{(\ell)}=\frac{q_{\ell}}{D_{1} D_{2}^{2} \hbar^{6}\left(B_{2}^{2}-8 D_{1}^{2}\right)^{2}}\left[D_{2}\left(B_{2}^{2}-8 D_{1}^{2}\right)\left(3 B_{2}^{2}\left(D_{1}^{2}+D_{2}^{2}\right)+8 D_{1}^{2}\left(2 D_{1}^{2}+D_{2}^{2}\right)\right) \hbar^{6}\right. \\
& \left.+\left(2 B_{2}^{3}\left(D_{1}^{3}+4 D_{2}^{2} D_{1}\right)-32 B_{2} D_{1}^{5}\right) \hbar^{4} q_{\ell}+D_{1}^{2} D_{2}\left(7 B_{2}^{2}+8 D_{1}^{2}\right) \hbar^{2} q_{\ell}^{2}+2 B_{2} D_{1}^{3} q_{\ell}^{3}\right], \\
& s_{4}^{(\ell)}=\frac{q_{\ell}}{D_{2}^{2} \hbar^{6}\left(B_{2}^{2}-8 D_{1}^{2}\right)^{2}}\left[4 B_{2} D_{2}\left(D_{1}^{2}+2 D_{2}^{2}\right)\left(B_{2}^{2}-8 D_{1}^{2}\right) \hbar^{6}\right. \\
& \left.+2 D_{1}\left(B_{2}^{2}\left(D_{1}^{2}+6 D_{2}^{2}\right)-16 D_{1}^{2}\left(D_{1}^{2}+D_{2}^{2}\right)\right) \hbar^{4} q_{\ell}+8 B_{2} D_{1}^{2} D_{2} \hbar^{2} q_{\ell}^{2}+2 D_{1}^{3} q_{\ell}^{3}\right], \\
& s_{5}=0
\end{aligned}
$$

where $q_{\ell}, \ell=1,2,3,4$, are the four roots of the quartic equation

$$
\begin{aligned}
& D_{1}^{4} q^{4}+4 B_{2} D_{2} D_{1}^{3} \hbar^{2} q^{3}+\left(B_{2}^{2}\left(D_{1}^{4}+6 D_{2}^{2} D_{1}^{2}\right)-16 D_{1}^{4}\left(D_{1}^{2}+D_{2}^{2}\right)\right) \hbar^{4} q^{2} \\
& -2 B_{2} D_{1}\left(8 D_{1}^{2}-B_{2}^{2}\right) D_{2}\left(D_{1}^{2}+2 D_{2}^{2}\right) \hbar^{6} q+\left(B_{2}^{2}-8 D_{1}^{2}\right)^{2} D_{2}^{2}\left(D_{1}^{2}+D_{2}^{2}\right) \hbar^{8}=0 .
\end{aligned}
$$

The discriminant $\Gamma$ of the quartic equation (44) is given by

$$
\Gamma=-256 \hbar^{24} D_{1}^{24} D_{2}^{2}\left(B_{2}^{2}-8 D_{1}^{2}\right)^{2}\left[B_{2}^{4}\left(60 D_{2}^{2}-48 D_{1}^{2}\right)+768 B_{2}^{2}\left(D_{1}^{2}+D_{2}^{2}\right)^{2}+B_{2}^{6}-4096\left(D_{1}^{2}+D_{2}^{2}\right)^{3}\right]
$$

This discriminant is zero if and only if at least two roots of (44) are equal. If the discriminant is negative there are two real roots and two complex conjugate roots. If it is positive the roots are either all real or all non-real. From a physical point of view only the real solutions are admitted. Let us analyze the zeros of the discriminant (45). They will correspond to the TTW model $\underline{46} \underline{47}$. For $\Gamma \neq 0$, by substituting (43) into (38) we obtain an angular component $S_{I}(\theta)$ proportional to $\hbar^{2}$ with no classical analog, it cannot be transformed or reduced to that of the TTW model. 
A.M. Escobar-Ruiz, J.C. López Vieyra, P. Winternitz and İ. Yurduşen

\section{Case $\hbar=0$}

For $\hbar=0$, the discriminant (45) vanishes. The corresponding coefficients take the values

$$
s_{1}=s_{1}, \quad s_{2}=0, \quad s_{3}=0, \quad s_{4}=s_{4}, \quad s_{5}=s_{5},
$$

which yields the potential

$$
S_{I}(\theta)=\frac{4\left(D_{1} \cos 4 \theta+D_{2} \sin 4 \theta\right) s_{1}+4\left(D_{1} s_{5}+D_{2} s_{4}\right)}{\left(D_{1}^{2}-D_{2}^{2}\right) \cos 8 \theta+2 D_{1} D_{2} \sin 8 \theta-\left(D_{1}^{2}+D_{2}^{2}\right)} .
$$

For $D_{2}=0$, the angular part of the potential becomes

$$
S_{I}(\theta)=\frac{\tilde{s}_{1} \cos 4 \theta+\tilde{s}_{5}}{\cos 8 \theta-1}
$$

where $\tilde{s}_{1}=4 s_{1} / D_{1}, \tilde{s}_{5}=4 s_{5} / D_{1}$. This potential (47) coincides with that of the TTW Hamiltonian $\underline{46}, \underline{47}$. The angular component of the TTW potential is

$$
\begin{aligned}
S_{\mathrm{TTW}}(\theta) & =\frac{\alpha k^{2}}{\cos ^{2}(k \theta)}+\frac{\beta k^{2}}{\sin ^{2}(k \theta)} \\
& =\frac{4 k^{2}(\alpha-\beta) \cos 2 k \theta-4 k^{2}(\alpha+\beta)}{\cos 4 k \theta-1} .
\end{aligned}
$$

Then for $k=2$ and $\alpha=\left(\tilde{s}_{1}-\tilde{s}_{5}\right) / 32, \beta=-\left(\tilde{s}_{1}+\tilde{s}_{5}\right) / 32$, the potential (47) actually corresponds to a rotated TTW model (with no radial component $R(r)=0$ ) which is a superintegrable system in both the classical and quantum cases.

\section{Case $D_{1}=0$}

For $D_{1}=0$, the discriminant (45) vanishes as well. The corresponding coefficients vanish, $s_{1}=s_{2}=s_{3}=s_{4}=s_{5}=0$, which gives the trivial solution $S_{I}(\theta)=0$.

\section{Case $D_{2}=0$}

For $D_{2}=0$, the discriminant (45) vanishes again. The corresponding coefficients are given by

$$
s_{1}=s_{1}, \quad s_{2}=0, \quad s_{3}=\frac{B_{2}^{2} s_{4}-8 D_{1}^{2}\left(s_{1}+s_{4}\right)}{2 B_{2} D_{1}}, \quad s_{4}=s_{4}, \quad s_{5}=0
$$


A.M. Escobar-Ruiz, J.C. López Vieyra, P. Winternitz and İ. Yurduşen

thus

$$
S(\theta)=-\frac{2\left(B_{2} s_{4}+2 D_{1} s_{1} \sin 2 \theta+2 D_{1} s_{4} \sin 2 \theta\right)}{B_{2} D_{1}(1+\cos 4 \theta)} .
$$

This solution corresponds to the angular component of the TTW model (48) with $k=1$. The zeros of the discriminant (45) correspond to a TTW model.

Case II: $\left(A_{3}, A_{4}, C_{1}, C_{2}\right) \neq(0,0,0,0)$

Now, we proceed to the case II. First, from the determining equation $\mathcal{C}_{r}=0$ (20) we obtain the function $G_{4}(r, \theta)$

$$
\begin{aligned}
48 r^{3} G_{4}(r, \theta) & =16 S^{\prime}\left[3 S^{\prime}\left(A_{4} \cos \theta-A_{3} \sin \theta-3\left(C_{2} \sin 3 \theta-C_{1} \cos 3 \theta\right)\right)\right. \\
& \left.+\left(S^{\prime \prime}-14 S\right)\left(A_{3} \cos \theta+A_{4} \sin \theta+C_{1} \sin 3 \theta+C_{2} \cos 3 \theta\right)\right] \\
& +\hbar^{2}\left[16 S\left(2\left(A_{4} \cos \theta-A_{3} \sin \theta\right)+15\left(C_{2} \sin 3 \theta-C_{1} \cos 3 \theta\right)\right)\right. \\
& 4 S^{\prime}\left(7\left(A_{3} \cos \theta+A_{4} \sin \theta\right)-127\left(C_{1} \sin 3 \theta+C_{2} \cos 3 \theta\right)\right) \\
& +4 S^{\prime \prime}\left(A_{3} \sin \theta-A_{4} \cos \theta+93\left(C_{1} \cos 3 \theta-C_{2} \sin 3 \theta\right)\right) \\
& +S^{(3)}\left(9\left(A_{3} \cos \theta+A_{4} \sin \theta\right)+121\left(C_{1} \sin 3 \theta+C_{2} \cos 3 \theta\right)\right) \\
& +6 S^{(4)}\left(A_{3} \sin \theta-A_{4} \cos \theta+3\left(C_{2} \sin 3 \theta-C_{1} \cos 3 \theta\right)\right) \\
& \left.-S^{(5)}\left(A_{3} \cos \theta+A_{4} \sin \theta+C_{1} \sin 3 \theta+C_{2} \cos 3 \theta\right)\right]
\end{aligned}
$$


A.M. Escobar-Ruiz, J.C. López Vieyra, P. Winternitz and İ. Yurduşen

and correspondingly $\mathcal{C}_{\theta}=0$ (21) takes the form

$$
\begin{aligned}
& 4\left[-36 S^{2}\left(A_{3} \cos \theta+A_{4} \sin \theta+C_{1} \sin 3 \theta+C_{2} \cos 3 \theta\right)\right. \\
& +15 S^{\prime 2}\left(A_{3} \cos \theta+A_{4} \sin \theta+9\left(C_{1} \sin 3 \theta+C_{2} \cos 3 \theta\right)\right) \\
& +20 S S^{\prime \prime}\left(A_{3} \cos \theta+A_{4} \sin \theta+C_{1} \sin 3 \theta+C_{2} \cos 3 \theta\right) \\
& -\left(S^{\prime \prime}\right)^{2}\left(A_{3} \cos \theta+A_{4} \sin \theta+C_{1} \sin 3 \theta+C_{2} \cos 3 \theta\right) \\
& +3 S^{\prime}\left(20 S\left(A_{4} \cos \theta-A_{3} \sin \theta+3\left(C_{1} \cos 3 \theta-C_{2} \sin 3 \theta\right)\right)\right. \\
& +5 S^{\prime \prime}\left(A_{3} \sin \theta-A_{4} \cos \theta+3\left(C_{2} \sin 3 \theta-C_{1} \cos 3 \theta\right)\right) \\
& \left.\left.-S^{(3)}\left(A_{3} \cos \theta+A_{4} \sin \theta+C_{1} \sin 3 \theta+C_{2} \cos 3 \theta\right)\right)\right] \\
& +\hbar^{2}\left[96 S\left(A_{3} \cos \theta+A_{4} \sin \theta+39\left(C_{1} \sin 3 \theta+C_{2} \cos 3 \theta\right)\right)\right. \\
& +24 S^{\prime}\left(A_{3} \sin \theta-A_{4} \cos \theta+303\left(C_{2} \sin 3 \theta-C_{1} \cos 3 \theta\right)\right) \\
& +8 S^{\prime \prime}\left(21\left(A_{3} \cos \theta+A_{4} \sin \theta\right)-719\left(C_{1} \sin 3 \theta+C_{2} \cos 3 \theta\right)\right) \\
& +30 S^{(3)}\left(3\left(A_{3} \sin \theta-A_{4} \cos \theta\right)+79\left(C_{1} \cos 3 \theta-C_{2} \sin 3 \theta\right)\right) \\
& +8 S^{(4)}\left(3\left(A_{3} \cos \theta+A_{4} \sin \theta\right)+67\left(C_{1} \sin 3 \theta+C_{2} \cos 3 \theta\right)\right) \\
& +21 S^{(5)}\left(A_{3} \sin \theta-A_{4} \cos \theta+3\left(C_{2} \sin 3 \theta-C_{1} \cos 3 \theta\right)\right) \\
& \left.-3 S^{(6)}\left(A_{3} \cos \theta+A_{4} \sin \theta+C_{1} \sin 3 \theta+C_{2} \cos 3 \theta\right)\right]=0
\end{aligned}
$$


Now we focus on the potential $S_{I I}(\theta)$ (40). By an appropriate rotation we can always set $A_{3}=0$. Then, we substitute the expression (40) into the remaining determining equation (42). As a result we obtain algebraic equations for the coefficients $s_{i}(i=1,2,3,4,5)$ by setting the coefficients of $\cos k \theta$ and $\sin k \theta$ for $k=1,2,3, \ldots, 16$ equal to zero. In general, we obtain the following three solutions

$$
\begin{aligned}
& s_{1}=0, \\
& s_{2}=s_{2}, \\
& s_{3}^{(\ell)}=q_{\ell}, \\
& s_{4}^{(\ell)}=\frac{\hbar^{4}\left(\left(2 C_{1}^{2}+C_{1} A_{4}+3 C_{2}^{2}\right) q_{\ell}+C_{1} C_{2} s_{2}\right)-\left(C_{1}+A_{4}\right) \hbar^{2} q_{\ell}^{2}-q_{\ell}^{3}}{A_{4} C_{2} \hbar^{4}}, \\
& s_{5}^{(\ell)}=\frac{\hbar^{4}\left(\left(4 C_{1}^{2}+C_{1} A_{4}+6 C_{2}^{2}-A_{4}^{2}\right) q_{\ell}+\left(2 C_{1}+A_{4}\right) C_{2} s_{2}\right)-\left(2 C_{1}+3 A_{4}\right) \hbar^{2} q_{\ell}^{2}-2 q_{\ell}^{3}}{A_{4}\left(2 C_{1}+A_{4}\right) \hbar^{4}},
\end{aligned}
$$

where $q_{\ell}, \ell=1,2,3$, is the solution to the cubic equation

$$
q^{3}+A_{4} \hbar^{2} q^{2}-\hbar^{4} q\left(3 C_{1}^{2}+2 A_{4} C_{1}+3 C_{2}^{2}\right)+\left(2 C_{1}^{3}+A_{4} C_{1}^{2}+2 C_{2}^{2} C_{1}+A_{4} C_{2}^{2}\right) \hbar^{6}=0 .
$$

Again, substituting (51) into (40) we obtain the corresponding angular component $S_{I I}(\theta)$ of the potential. Notice that there exist potentials $S_{I I}(\theta)$ proportional to $\hbar^{2}$ that have no classical analog.

The discriminant $\Omega$ of the cubic equation (52) is given by

$$
\Omega=-4 \hbar^{12} C_{2}^{2}\left[A_{4}^{4}+8 A_{4}^{3} C_{1}+18 A_{4}^{2}\left(C_{1}^{2}+C_{2}^{2}\right)-27\left(C_{1}^{2}+C_{2}^{2}\right)^{2}\right]
$$

The discriminant (53) is zero if and only if at least two roots are equal. It is positive if the roots are all distinct real numbers, and negative if there exist one real and two complex conjugate roots.

For $\hbar=0$, the discriminant (53) vanishes. The corresponding coefficients take the values

$$
s_{1}=s_{1}, \quad s_{2}=0, \quad s_{3}=0, \quad s_{4}=s_{4}, \quad s_{5}=s_{5},
$$

which yields the potential

$$
S_{I I}(\theta)=-\frac{6\left(s_{5}+s_{1} \cos 3 \theta\right)}{C_{1}(1-\cos 6 \theta)}
$$


$\left(A_{4}=C_{2}=0\right)$ it corresponds to that of the TTW potential with $k=\frac{3}{2}$. Similarly we can show that for $C_{2}=0$, thus $\Omega=0$, the TTW (angular) potentials with $k=\frac{1}{2}$ and $k=1$ occur.

\section{CONFINING POTENTIALS}

\section{Deformed Coulomb Potential}

Here we address the case of the deformed Coulomb potential

$$
V(r, \theta)=\frac{a}{r}+\frac{S(\theta)}{r^{2}}
$$

where $a \neq 0$ is a real constant.

Similarly to the non confining case $R(r)=0$, from (16), (18) and (19) corresponding to the determining equations $\mathcal{A}_{r r r}=\mathcal{A}_{r r \theta}=\mathcal{A}_{r \theta \theta}=0$, respectively, we determine the functions $G_{1}(r, \theta), G_{3}(r, \theta)$, and $G_{2}(r, \theta)$ up to arbitrary additive functions $\gamma_{1}(\theta), \gamma_{3}(\theta)$ and $\gamma_{2}(\theta)$, respectively.

Then, we substitute such functions $G_{1,2,3}$ into the determining equation $\mathcal{A}_{\theta \theta \theta}=0$ (17). By doing so, such equation yields again a set of five linear ODE

- 1. Two fourth-order ODE for the angular component $S(\theta)$. The first ODE contains the doublets $\left(B_{1}, B_{2}\right),\left(D_{1}, D_{2}\right)$, while the second ones depends on the doublets $\left(A_{3}, A_{4}\right),\left(C_{1}, C_{2}\right)$ and the parameter $a$ figuring in the Coulomb term.

- 2. Three linear ODE that define the functions $\gamma_{1}(\theta), \gamma_{2}(\theta)$ and $\gamma_{3}(\theta)$, respectively. The equation for $\gamma_{1}$ depends on the doublet $\left(B_{3}, B_{4}\right)$ and the parameter $a$. For the function $\gamma_{3}$ the doublet $\left(A_{1}, A_{2}\right)$ and the parameter $a$ are involved and the equation for $\gamma_{2}$ contains only the parameter $a$.

For $a \neq 0$ the most general form for the angular component $S(\theta)$ is given by the Case II (40) for which at least one of the constants $C_{1}, C_{2}, A_{3}, A_{4}$ is not vanishing. Therefore, the function $S(\theta)$ is not new with respect to the non-confining case $R(r)=0$ and it was already described in detail in the Section โII. 
The corresponding functions $G_{1,2,3}$ are given by

$$
\begin{aligned}
& G_{1}(r, \theta)=G_{1}^{(0)}(r, \theta), \\
& G_{2}(r, \theta)=G_{2}^{(0)}(r, \theta)+5 a\left[\frac{A_{4} \cos \theta-A_{3} \sin \theta+3\left(C_{1} \cos 3 \theta-C_{2} \sin 3 \theta\right)}{2 r^{2}}\right], \\
& G_{3}(r, \theta)=G_{3}^{(0)}(r, \theta)+3 a\left[\frac{A_{3} \cos \theta+A_{4} \sin \theta+C_{2} \cos 3 \theta+C_{1} \sin 3 \theta}{r}\right],
\end{aligned}
$$

where the $G_{i}^{(0)}(r, \theta), i=1,2,3$, coincide with those of the non-confining potential given by (27), (28) and (29), respectively, putting $B_{1}=B_{2}=D_{1}=D_{2}=0$ and $\gamma_{1}=\gamma_{2}=\gamma_{3}=0$.

From the determining equation $\mathcal{C}_{r}=0$ given in (20) we calculate the corresponding function $G_{4}(r, \theta)$

$$
\begin{aligned}
G_{4}(r, \theta) & =G_{4}^{(0)}(r, \theta)+a\left[\frac { 1 } { r ^ { 2 } } \left(\frac{1}{4} \hbar^{2}\left(7\left(A_{4} \cos \theta-A_{3} \sin \theta\right)+99\left(C_{2} \sin 3 \theta-C_{1} \cos 3 \theta\right)\right)\right.\right. \\
& \left.+5 S^{\prime}(\theta)\left(A_{4} \sin \theta+A_{3} \cos \theta+C_{1} \sin 3 \theta+C_{2} \cos 3 \theta\right)\right)
\end{aligned}
$$

here $G_{4}^{(0)}(r, \theta)$ is given in (49). The angular component $S(\theta)$ is given by (40) with the coefficients $s_{i}(\underline{51})$ presented in the Case II.

\section{Deformed Harmonic Oscillator Potential}

Here we address the case of the confining potential

$$
V(r, \theta)=b r^{2}+\frac{S(\theta)}{r^{2}}
$$

with $b \neq 0$ a real constant.

Following the same strategy as for the previous case, we obtain the following functions

$$
\begin{aligned}
& G_{1}(r, \theta)=G_{1}^{(0)}(r, \theta)+2 b r^{2}\left(B_{1} \cos 2 \theta+B_{2} \sin 2 \theta+D_{1} \cos 4 \theta+D_{2} \sin 4 \theta\right), \\
& G_{2}(r, \theta)=G_{2}^{(0)}(r, \theta), \\
& G_{3}(r, \theta)=G_{3}^{(0)}(r, \theta)+2 b r\left(B_{2} \cos 2 \theta-B_{1} \sin 2 \theta+2\left(D_{2} \cos 4 \theta-D_{1} \sin 4 \theta\right)\right), \\
& G_{4}(r, \theta)=G_{4}^{(0)}(r, \theta),
\end{aligned}
$$

where the $G_{i}^{(0)}(r, \theta), i=1,2,3$, coincide with those of the non-confining potential $R(r)=0$, given in (27), (28) and (29), respectively, putting $A_{3}=A_{4}=C_{1}=C_{2}=0$ and $\gamma_{1}=\gamma_{2}=$ $\gamma_{3}=0$. The function $G_{4}^{(0)}(r, \theta)$ is given in (41) $)$.

For $b \neq 0$, the angular component $S(\theta)$ is given by (38) with the coefficients $s_{i}$ (43) presented in the Case I for which at least one of the constants $B_{1}, B_{2}, D_{1}, D_{2}$ is not vanishing. 
A.M. Escobar-Ruiz, J.C. López Vieyra, P. Winternitz and İ. Yurduşen

\section{CONCLUSIONS}

We considered superintegrable systems in a two-dimensional Euclidean space. Classical and quantum fourth-order superintegrable standard potentials separating in polar coordinates were derived. We can summarize the main results via the following theorems

Theorem 1. In classical mechanics, the standard superintegrable confining systems, $a b \neq 0$, correspond to the TTW potential

$$
V_{\mathrm{TTW}}(r, \theta)=b r^{2}+\frac{1}{r^{2}}\left[\frac{\alpha}{\cos ^{2} 2 \theta}+\frac{\beta}{\sin ^{2} 2 \theta}\right],
$$

( $\alpha, \beta$ real constants), and the $P W$ potential

$$
V_{\mathrm{PW}}(r, \theta)=\frac{a}{r}+\frac{1}{r^{2}}\left[\frac{\mu}{\cos ^{2} \frac{3}{2} \theta}+\frac{\nu}{\sin ^{2} \frac{3}{2} \theta}\right],
$$

$(\mu, \nu$ real constants). The corresponding leading terms of the integral $Y$ in (9) are proportional to

$$
\left(p_{x}^{4}+p_{y}^{4}-6 p_{x}^{2} p_{y}^{2}\right)
$$

and

$$
\left\{L_{z}, 3 p_{x}^{2} p_{y}-p_{y}^{3}\right\}
$$

respectively. These terms are independent of $a$ and $b$.

Theorem 2. In quantum mechanics, the new confining superintegrable systems correspond to

$$
V(r, \theta)=b r^{2}+\frac{1}{r^{2}} S_{I}(\theta)
$$

and

$$
V(r, \theta)=\frac{a}{r}+\frac{1}{r^{2}} S_{I I}(\theta)
$$

where $S_{I}(\theta)$ is given by (38) with (43), and $S_{I I}(\theta)$ takes the form (40) with (51). In general, the functions $S_{I}$ and $S_{I I}$ are proportional to $\hbar^{2}$ and cannot be reduced to a TTW or PW potential. The corresponding leading terms of the integral $Y$ in (9) are

$$
2 B_{2} p_{x} p_{y}\left(p_{x}^{2}+p_{y}^{2}\right)+D_{1}\left(p_{x}^{4}+p_{y}^{4}-6 p_{x}^{2} p_{y}^{2}\right)+4 D_{2} p_{x} p_{y}\left(p_{x}^{2}-p_{y}^{2}\right)
$$

$\left(B_{2}, D_{1}, D_{2}\right) \neq(0,0,0)$ and

$$
A_{4}\left\{L_{z}, p_{y}\left(p_{x}^{2}+p_{y}^{2}\right)\right\}+C_{1}\left\{L_{z}, 3 p_{x}^{2} p_{y}-p_{y}^{3}\right\}+C_{2}\left\{L_{z}, p_{x}^{3}-3 p_{y}^{2} p_{x}\right\}
$$


$\left(A_{4}, C_{1}, C_{2}\right) \neq(0,0,0)$ respectively

We emphasize that these are pure quantum potentials. As particular cases, both potentials $V_{\mathrm{TTW}}$ and $V_{\mathrm{PW}}$ appear in the quantum case as well.

Work is currently in progress on a general and unified study of $N^{\text {th }}$-order exotic and standard potentials separating in polar coordinates 13 . Within this study $\underline{13}$ the TTW and PW models correspond to standard classical potentials. For some cases we plan to present the polynomial algebra generated by the integrals of motion and to use it to calculate the energy spectrum and the wave functions in the quantum case.

\section{ACKNOWLEDGMENTS}

The research of PW was partially supported by a research grant from NSERC of Canada. JCLV thanks PASPA grant (UNAM, Mexico) and the Centre de Recherches Mathématiques, Université de Montréal for the kind hospitality while on sabbatical leave during which this work was done. The research of AME was partially supported by a fellowship awarded by the Laboratory of Mathematical Physics of the CRM. The research of IY was partly supported by Hacettepe University Scientific Research Coordination Unit. Project Number: FBI-2017-14035. He also thanks the Centre de Recherches Mathématiques, Université de Montréal and especially Professor Pavel Winternitz for kind hospitality during his sabbatical leave. 


\section{APPENDIX}

Explicitly, the functions $F_{1}, \ldots, F_{13}$ figuring in (16)-(21) are given by

$$
\begin{aligned}
& F_{1}=2\left(B_{1} \cos 2 \theta+B_{2} \sin 2 \theta+D_{1} \cos 4 \theta+D_{2} \sin 4 \theta\right), \\
& F_{2}=\frac{1}{r}\left(B_{2} \cos 2 \theta-B_{1} \sin 2 \theta-2\left(D_{1} \sin 4 \theta-D_{2} \cos 4 \theta\right)\right) \\
& +A_{3} \cos \theta+A_{4} \sin \theta+C_{1} \sin 3 \theta+C_{2} \cos 3 \theta, \\
& F_{3}=\frac{1}{r^{3}}\left(B_{2} \cos 2 \theta-B_{1} \sin 2 \theta+2\left(D_{1} \sin 4 \theta-D_{2} \cos 4 \theta\right)\right) \\
& +\frac{1}{r^{2}}\left(A_{4} \sin \theta+A_{3} \cos \theta-3\left(C_{2} \cos 3 \theta+C_{1} \sin 3 \theta\right)\right) \\
& -\frac{2}{r}\left(B_{3} \sin 2 \theta-B_{4} \cos 2 \theta\right)+A_{2} \sin \theta+A_{1} \cos \theta, \\
& F_{4}=\frac{2}{r^{4}}\left(D_{1} \cos 4 \theta+D_{2} \sin 4 \theta-B_{1} \cos 2 \theta-B_{2} \sin 2 \theta\right) \\
& +\frac{4}{r^{3}}\left(A_{4} \cos \theta-A_{3} \sin \theta-C_{1} \cos 3 \theta+C_{2} \sin 3 \theta\right) \\
& -\frac{4}{r^{2}}\left(B_{3} \cos 2 \theta+B_{4} \sin 2 \theta\right)+\frac{4}{r}\left(A_{2} \cos \theta-A_{1} \sin \theta\right), \\
& F_{5}=-\frac{6}{r^{2}}\left(D_{1} \cos 4 \theta+D_{2} \sin 4 \theta\right)+\frac{2}{r}\left(A_{4} \cos \theta-A_{3} \sin \theta\right. \\
& \left.+3\left(C_{1} \cos 3 \theta-C_{2} \sin 3 \theta\right)\right)+2\left(B_{3} \cos 2 \theta+B_{4} \sin 2 \theta\right), \\
& F_{6}=\frac{3}{2}\left(A_{4} \cos \theta-A_{3} \sin \theta+3\left(C_{1} \cos 3 \theta-C_{2} \sin 3 \theta\right)\right)-\frac{9}{r}\left(D_{1} \cos 4 \theta+D_{2} \sin 4 \theta\right), \\
& F_{7}=\frac{12}{r^{2}}\left(D_{1} \sin 4 \theta-D_{2} \cos 4 \theta\right)+\frac{1}{2 r}\left(A_{4} \sin \theta+A_{3} \cos \theta\right. \\
& \left.-15\left(C_{1} \sin 3 \theta+C_{2} \cos 3 \theta\right)\right)-2\left(B_{3} \sin 2 \theta-B_{4} \cos 2 \theta\right), \\
& F_{8}=-\frac{3}{r^{3}}\left(B_{1} \cos 2 \theta+B_{2} \sin 2 \theta-5\left(D_{1} \cos 4 \theta+D_{2} \sin 4 \theta\right)\right) \\
& +\frac{3}{2 r^{2}}\left(A_{4} \cos \theta-A_{3} \sin \theta-9\left(C_{1} \cos 3 \theta-C_{2} \sin 3 \theta\right)\right) \\
& -\frac{5}{r}\left(B_{3} \cos 2 \theta+B_{4} \sin 2 \theta\right)+\frac{3}{2}\left(A_{2} \cos \theta-A_{1} \sin \theta\right), \\
& F_{9}=\frac{9}{r^{4}}\left(B_{1} \sin 2 \theta-B_{2} \cos 2 \theta-2\left(D_{1} \sin 4 \theta-D_{2} \cos 4 \theta\right)\right) \\
& +\frac{15}{2 r^{3}}\left(3\left(C_{1} \sin 3 \theta+C_{2} \cos 3 \theta\right)-A_{3} \cos \theta-A_{4} \sin \theta\right) \\
& +\frac{12}{r^{2}}\left(B_{3} \sin 2 \theta-B_{4} \cos 2 \theta\right)-\frac{9}{2 r}\left(A_{1} \cos \theta+A_{2} \sin \theta\right), \\
& F_{10}=\frac{3}{r}\left(\left(B_{2} \cos 2 \theta-B_{1} \sin 2 \theta\right)+2\left(D_{2} \cos 4 \theta-D_{1} \sin 4 \theta\right)\right) \\
& \left.+3\left(A_{3} \cos \theta+A_{4} \sin \theta+C_{1} \sin 3 \theta+C_{2} \cos 3 \theta\right)\right), \\
& F_{11}=-\frac{3}{r^{2}}\left(B_{1} \cos 2 \theta+B_{2} \sin 2 \theta-5\left(D_{1} \cos 4 \theta+D_{2} \sin 4 \theta\right)\right)-4\left(B_{3} \cos 2 \theta+B_{4} \sin 2 \theta\right) \\
& +\frac{1}{r}\left(A_{4} \cos \theta-A_{3} \sin \theta-9\left(C_{1} \cos 3 \theta 26 C_{2} \sin 3 \theta\right)\right),
\end{aligned}
$$


A.M. Escobar-Ruiz, J.C. López Vieyra, P. Winternitz and İ. Yurduşen

$$
\begin{aligned}
F_{12} & =\frac{2}{r^{3}}\left(B_{1} \sin 2 \theta-B_{2} \cos 2 \theta-14\left(D_{1} \sin 4 \theta-D_{2} \cos 4 \theta\right)\right) \\
& +\frac{3}{2 r^{2}}\left(11\left(C_{1} \sin 3 \theta+C_{2} \cos 3 \theta\right)-A_{3} \cos \theta-A_{4} \sin \theta\right) \\
& +\frac{6}{r}\left(B_{3} \sin 2 \theta-B_{4} \cos 2 \theta\right)-\frac{3}{2}\left(A_{1} \cos \theta+A_{2} \sin \theta\right), \\
F_{13} & =\frac{4}{r^{4}}\left(2\left(B_{1} \cos 2 \theta+B_{2} \sin 2 \theta\right)-11\left(D_{1} \cos 4 \theta+D_{2} \sin 4 \theta\right)\right) \\
& -\frac{2}{r^{3}}\left(A_{4} \cos \theta-A_{3} \sin \theta-17\left(C_{1} \cos 3 \theta-C_{2} \sin 3 \theta\right)\right) \\
& +\frac{12}{r^{2}}\left(B_{3} \cos 2 \theta+B_{4} \sin 2 \theta\right)-\frac{3}{r}\left(A_{2} \cos \theta-A_{1} \sin \theta\right) .
\end{aligned}
$$

\section{REFERENCES}

${ }^{1}$ Abouamal I and Winternitz P 2018 Fifth-order superintergrable quantum system separating in Cartesian coordinates: Doubly exotic potentials J. Math. Phys. 59022104

${ }^{2}$ Bermudez D, Fernández C D J and Negro J 2016 Solutions to the Painlevé V equation through supersymmetric quantum mechanics J. Phys. A-Math. Theor. 49335203

${ }^{3}$ Bertrand J L F 1873 Théorème relatif au mouvement d'un point attiré vers un centre fixe C. R. Acad. Sci. $77849-853$

${ }^{4}$ Boyer C P, Kalnins E G and Miller W Jr 1986 Stäckel-equivalent integrable Hamiltonian systems SIAM J. Math. Anal. 17 778-797

${ }^{5}$ Capel J J, Kress J M and Post S 2015 Invariant classification and limits of maximally superintegrable systems in 3D SIGMA 1117

${ }^{6}$ Cariñena J F, Herranz F J and Rañada M F 2017 Superintegrable systems on 3-dimensional curved spaces: Eisenhart formalism and separability J. Math. Phys. 58022701

${ }^{7}$ Celeghini E, Kuru Ş, Negro J and del Olmo M A 2013 A unified approach to quantum and classical TTW systems based on factorizations Ann. Phys. 332 27-37

${ }^{8}$ Chanu C, Degiovanni L Rastelli G 2011 First integrals of extended Hamiltonians in n+1 dimensions generated by powers of an operator SIGMA 712

${ }^{9}$ Chanu C, Degiovanni L Rastelli G 2012 Superintegrable extensions of superintegrable systems SIGMA 812

${ }^{10}$ Drach J 1935 Sur l'integration logique des équations de la dynamique à deux variables: Forces constructives. Intégrales cubiques. Mouvements dans le plan Comptes Rendus Acad.

Sci. 20022 
A.M. Escobar-Ruiz, J.C. López Vieyra, P. Winternitz and İ. Yurduşen

${ }^{11}$ Drach J 1935 Sur l'integration logique et sur la tranformation des équations de la dynamique à deux variables: Forces conservatives. Intégrales cubiques. Mouvements dans le plan Comptes Rendus Acad. Sci. 200599

${ }^{12}$ Escobar-Ruiz A M, López Vieyra J C and Winternitz P 2018 Fourth order superintegrable systems separating in Polar coordinates. I. Exotic Potentials J. Phys. A-Math. Theor. 50 495206

${ }^{13}$ Escobar-Ruiz A M, Winternitz P and Yurduşen İ General $N^{\text {th }}$-order superintegrable systems separating in Polar coordinates (in preparation)

${ }^{14}$ Fernández C D J and Morales-Salgado V S 2016 SUSY partners of the truncated oscillator, Painlevé transcendents and Bäcklund transformations J. Phys. A-Math. Theor. 49195202

${ }^{15}$ Friš J, Mandrosov V, Smorodinsky Y A, Uhlír M and Winternitz P 1965 On higher symmetries in quantum mechanics Phys. Lett. 16 354-6

${ }^{16}$ Friš J, Smorodinsky Y A, Uhlî́r M and Winternitz P 1966 Symmetry groups in classical and quantum mechanics Yadra Fiz. 4 625-35

${ }^{17}$ Gonera C 2012 On the superintegrability of the TTW model Phys. Lett. A 376 2341-3

${ }^{18}$ Gravel S 2004 Hamiltonians separable in Cartesian coordinates and third-order integrals of motion J. Math. Phys. 45 1003-19

${ }^{19}$ Gravel S and Winternitz P 2002 Superintegrability with third-order integrals in quantum and classical mechanics J. Math. Phys. 43 5902-12

${ }^{20}$ Güngör F, Kuru Ş, Negro J and Nieto L M 2017 Heisenberg-type higher order symmetries of superintegrable systems separable in cartesian coordinates Nonlinearity 30 1788-808

${ }^{21}$ Hakobyan T, Nersessian A and Shmavonyan H 2017 Lobachevsky geometry in TTW and PW systems Phys. Atomic Nuclei 80 598-604

${ }^{22}$ Hietarinta J 1998 Pure quantum integrability Phys. Lett. A 246 97-104

${ }^{23}$ Hietarinta J, Grammaticos B, Dorizzi B and Ramani A 1984 Coupling-constant metamorphosis and duality between integrable Hamiltonian systems Phys. Rev. Lett. 53 1707-10

${ }^{24}$ Kalnins E G, Kress J M and Miller W Jr 2005 Second-order superintegrable systems in conformally flat spaces. I. Two-dimensional classical structure theory J. Math. Phys. 46 053509

${ }^{25}$ Kalnins E G, Kress J M and Miller W Jr 2005 Second-order superintegrable systems in conformally flat spaces. II. The classical two-dimensional Stäckel transform J. Math. Phys. 46053510 
${ }^{26}$ Kalnins E G, Kress J M and Miller W Jr 2005 Second order superintegrable systems in conformally flat spaces. III. Three-dimensional classical structure theory J. Math. Phys. 46103507

${ }^{27}$ Kalnins E G, Kress J M and Miller W Jr 2006 Second order superintegrable systems in conformally flat spaces. IV. The classical 3D Stäckel transform and 3D classification theory J. Math. Phys. 47043514

${ }^{28}$ Kalnins E G, Kress J M and Miller W Jr 2006 Second-order superintegrable systems in conformally flat spaces. V. Two- and three-dimensional quantum systems J. Math. Phys. 47093501

${ }^{29}$ Kalnins E G, Kress J M and Miller W Jr 2010 Superintegrability and higher order integrals for quantum systems J. Phys. A-Math. Theor. 43265205

${ }^{30}$ Kalnins E G, Miller W Jr, Pogosyan G S 2011 Superintegrability and higher-order constants for classical and quantum systems Phys. Atomic Nuclei 74 914-8

${ }^{31}$ Kalnins E G, Miller W Jr and Winternitz P 1976 The Group O(4), Separation of Variables and the Hydrogen Atom SIAM J. Appl. Math. 30 630-64

${ }^{32}$ Kalnins E G, Miller W Jr, Post S 2010 Coupling constant metamorphosis and $N^{\text {th }}$-order symmetries in classical and quantum mechanics J. Phys. A-Math. Theor. 43035202

${ }^{33}$ Kalnins E G, Williams G C, Miller W Jr and Pogosyan G S 2002 On superintegrable symmetry-breaking potentials in N-dimensional Euclidean space J. Phys. A-Math. Gen. $354755-73$

${ }^{34}$ Makarov A A, Smorodinsky J A, Valiev K and Winternitz P 1967 A systematic search for nonrelativistic systems with dynamical symmetries Nuovo Cimento A 52 1061-84

${ }^{35}$ Marchesiello A, Post S and Šnobl L 2015 Third-order superintegrable systems with potentials satisfying only nonlinear equations J. Math. Phys. 56102104

${ }^{36}$ Marquette I 2009 Superintegrability with third order integrals of motion, cubic algebras, and supersymmetric quantum mechanics. II. Painlevé transcendent potentials J. Math. Phys. 50 095202-2

${ }^{37}$ Marquette I 2010 Superintegrability and higher order polynomial algebras J. Phys. AMath. Gen. 43135203

${ }^{38}$ Marquette I, Sajedi M and Winternitz P 2017 Fourth order Superintegrable systems separating in Cartesian coordinates I. Exotic quantum potentials J. Phys. A-Math. Theor. 50 315201 
${ }^{39}$ Miller W Jr, Post S and Winternitz P 2013 Classical and quantum superintegrability with applications J. Phys. A-Math. Gen. 46423001

${ }^{40}$ Popper I, Post S and Winternitz P 2012 Third-order superintegrable systems separable in parabolic coordinates J. Math. Phys. 53062105

${ }^{41}$ Post S, Vinet L and Zhedanov A 2011 An infinite family of superintegrable Hamiltonians with reflection in the plane J. Phys. A-Math. Theor. 44505201

${ }^{42}$ Post S and Winternitz P 2010 An infinite family of superintegrable deformations of the Coulomb potential J. Phys. A-Math. Gen. 4322201

${ }^{43}$ Post S and Winternitz P 2015 General $N^{\text {th }}$-order integrals of motion in the Euclidean plane J. Phys. A-Math. Theor. 48405201

${ }^{44}$ Quesne C 2010 Superintegrability of the Tremblay-Turbiner-Winternitz quantum Hamiltonians on a plane for odd k J. Phys. A-Math. Theor. 43082001

${ }^{45}$ Rañada M F 2013 Higher order superintegrability of separable potentials with a new approach to the Post-Winternitz system J. Phys. A-Math. Theor. 46125206

${ }^{46}$ Tremblay F, Turbiner A V and Winternitz P 2009 An infinite family of solvable and integrable quantum systems on a plane J. Phys. A-Math. Theor. 42242001

${ }^{47}$ Tremblay F, Turbiner A V and Winternitz P 2010 Periodic orbits for an infinite family of classical superintegrable systems J. Phys. A-Math. Theor. 43015202

${ }^{48}$ Tremblay F and Winternitz P 2010 Third-order superintegrable systems separating in polar coordinates J. Phys. A-Math. Theor. 43175206

${ }^{49}$ Tsiganov A V 2000 The Drach superintegrable systems J. Phys. A-Math. Gen. 337407 